\title{
ENTENDENDO ESTRUTURA MOLECULAR COM A MOLÉCULA DE HIDROGÊNIO IONIZADA
}

\author{
Roberto Rivelino de Melo Moreno* \\ Instituto de Física - Universidade Federal da Bahia - 40210-340 - Salvador - BA \\ Leonardo Sena Gomes Teixeira
}

Instituto de Química - Universidade Federal da Bahia - 40170-290 - Salvador - BA

Recebido em 31/8/98; aceito em 4/1/99

\begin{abstract}
UNDERSTANDING THE MOLECULAR STRUCTURE USING THE IONIZED HYDROGEN MOLECULE. In this paper a general view about the modern molecular structure theory is developed discussing the ionized hydrogen molecule. We introduce some necessary approximation methods for the electronic and nuclear spectra study adopting a systematic approach. In addition though, we have performed calculations in order to illustrate these methods.
\end{abstract}

Keywords: molecular structure theory; ionized hydrogen molecule; approximation methods.

\section{INTRODUÇÃO}

A hipótese da teoria de estrutura molecular - de que as moléculas são formadas a partir de átomos, ligados por forças interatômicas e originando espécies estáveis - foi um dos maiores avanços realizados pelos químicos do século XIX e constitui, hoje, a base fundamental para a compreensão de grande parte dos fenômenos químicos ${ }^{1,2}$. Entretanto, sem o desenvolvimento da teoria quântica não seria possível responder questões cruciais, relacionadas à moderna linguagem da teoria de estrutura da matéria. Por que os átomos se combinam em número definido para formar uma molécula? Por que as moléculas assumem determinadas formas geométricas? Como os átomos podem se agrupar, originando moléculas estáveis? Por que os espectros moleculares vão desde a faixa de micro-ondas até a faixa do ultravioleta? Estas são algumas das perguntas discutidas entre físicos e químicos, as quais só obtiveram respostas satisfatórias à luz da teoria quântica. Contudo, não podemos afirmar que esta descrição da estrutura molecular é assunto encerrado na química.

Uma das aplicações mais importantes da teoria quântica na química foi a realização de cálculos relacionados à estrutura de átomos e moléculas simples nas décadas de 20 e $30^{3-11}$. Historicamente, o estudo mecânico-quântico do átomo de hidrogênio foi crucial para a compreensão posterior dos átomos multi-eletrônicos ${ }^{12-14}$. Da mesma maneira, o estudo mecânicoquântico dos espectros de energia da molécula de hidrogênio ionizada $^{10-12,15}$ teve fundamental importância para o desenvolvimento das modernas teorias de estrutura molecular. A molécula de hidrogênio ionizada foi detectada pela primeira vez por J. J. Thomson em seus experimentos de tubos de raios catódicos ${ }^{16}$. Embora esta molécula seja considerada um sistema muito simples, o tratamento quântico de seus espectros fornece uma importante base para a compreensão da estrutura eletrônica de moléculas poliatômicas.

Nesta comunicação, procura-se estudar alguns aspectos teóricos sobre estrutura eletrônica molecular, dando ênfase à teoria do orbital molecular ${ }^{2,16-18}$, a partir do estudo da molécula de hidrogênio ionizada. Para isto, utilizou-se o modelo de dois prótons e um elétron interagindo entre si por forças eletromagnéticas, e que se movem segundo as leis da mecânica

E-mail: rivelino@ufba.br

E-mail: 1sgt@ufba.br quântica $^{12,16}$. As etapas necessárias para resolver os espectros de energia desta molécula foram estudadas separadamente e, sempre que possível, estendeu-se os resultados para moléculas mais complexas. Espera-se, assim, mostrar um caminho didático para uma melhor compreensão desta teoria tão importante na química moderna, por parte de estudantes de graduação, pós-graduação e profissionais ligados ou não a área de química quântica.

\section{O MODELO DA MOLÉCULA DE HIDROGÊNIO IONIZADA}

$\mathrm{O}$ modelo da molécula de hidrogênio ionizada, $\mathrm{H}_{2}^{+}$, é mostrada no sistema de coordenadas da Figura 1: um elétron e dois prótons interagindo eletricamente entre si via forças coulombianas e cuja dinâmica é descrita de acordo com a mecânica quântica.

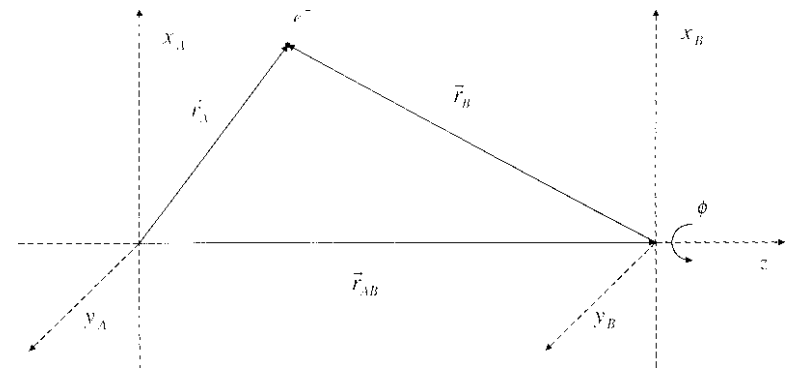

Figura 1. Sistema de referência para a molécula de hidrogênio ionizada. Os núcleos $A$ e $B$ estão separados pelo vetor $\vec{r}_{A B}$, e o elétron, representado pelo símbolo é, está localizado em relação aos dois prótons pelos vetores $\dot{r}_{A}, \grave{r}_{B}$ e pelo ângulo $\phi$, em torno do eixo $z$.

Para discutirmos os espectros de energia de uma molécula é necessário definir seu operador ${ }^{*}$ Hamiltoniano, $\hat{H}$, observável responsável pela dinâmica do sistema ${ }^{12-18}$. No caso do $\mathrm{H}_{2}{ }^{+}$, temos

$\hat{H}=\hat{T}_{A}+\hat{T}_{B}+\hat{T}_{\varepsilon}+\hat{V}_{A B}+\hat{V}_{A \varepsilon}+\hat{V}_{B \varepsilon}$

Em mecânica quântica todos os observáveis físicos são representados por operadores (ver, por exemplo, P. A. M. Dirac, em The Principles of Quantum Mechanics, 1958). 
onde os termos $\hat{T}$ e $\hat{V}$ representam, respectivamente, os operadores de energias cinética e potencial. Os índices $A$ e $B$ designam os núcleos e o índice $\varepsilon$ refere-se ao elétron. Explicitando estes termos ${ }^{19}$, tem-se:

$\hat{T}_{P}=-\frac{\mathrm{h}^{2}}{2 m_{P}} \nabla_{P}^{2}, P=A, B$

$\hat{T}_{\varepsilon}=-\frac{h^{2}}{2 m_{\varepsilon}} \nabla_{\varepsilon}^{2}$

$\hat{V}_{P \varepsilon}=-\frac{e^{2}}{r_{P}},{ }_{P=A, B}$

$\hat{V}_{A B}=\frac{e^{2}}{r_{A B}}$

sendo $m_{p}$ a massa do próton, $m_{\varepsilon}$ a massa do elétron, e $e$ a carga elementar.

A nossa proposta é estudar os estados estacionários da molécula de $\mathrm{H}_{2}{ }^{+}$. Estes estados são encontrados a partir da solução da seguinte equação de autovalor ${ }^{16,19}$

$\hat{H} \Psi_{n, v}=E_{n, v} \Psi_{n, v}$

onde $\Psi_{n, v}=\Psi_{n, v}\left(\dot{r}_{A}, \stackrel{1}{r}_{B}, \stackrel{t}{A B}_{A B}\right)$ são as funções de estado da molécula associadas aos valores de energia $E_{n, v}$. Devemos notar que o problema do $\mathrm{H}_{2}{ }^{+}$é um problema de três $\operatorname{corpos}^{12,16}$, e portanto, insolúvel em sua forma geral. Entretanto, é possível realizar aproximações adequadas que tornam a eq. (3) solúvel.

O primeiro nível de aproximação que utilizaremos para resolver a eq. (3) é a separação de Born-Oppenheimer, a qual supõe que os núcleos, sendo mais pesados que os elétrons, movem-se mais lentamente em relação a estes, e por isto podem ser tratados como se fossem fixos ${ }^{19}$. Assim, torna-se possível desacoplar o movimento eletrônico do movimento dos núcleos, o que significa escrever a função de estado da molécula, $\Psi_{n, v}\left(r_{A}, \stackrel{r}{r}_{B}, r_{A B}\right)$ como um produto de uma função de estado eletrônica, $\psi_{n}\left(\dot{r}_{A B} ; \dot{r}_{A} ; \dot{r}_{B}\right)$, para uma dada configuração dos núcleos, $\dot{r}_{A B}$, e de uma função de estado nuclear, $X_{n, v}\left(r_{A B}\right)$. Partindo-se desta aproximação podemos reescrever a eq. (3) em duas partes:

$\hat{H}_{\varepsilon} \psi_{n}\left(\stackrel{r}{r_{A B}} ; \stackrel{r}{r_{A}}, \underset{r}{r_{B}}\right)=W_{n} \psi_{n}\left(\stackrel{r}{r_{A B}} ; \stackrel{r}{r_{A}}, \underset{r}{r_{B}}\right)$

$\hat{H}_{N} X_{n, v}\left(\stackrel{r}{r_{A B}}\right)=E_{n, v} X_{n, v}\left(\stackrel{r}{r_{A B}}\right)$

A eq. (4) descreve o movimento eletrônico no campo eletrostático dos núcleos, com o operador Hamiltoniano dado por

$\hat{H}_{\varepsilon}=\hat{T}_{\varepsilon}+\hat{V}_{A \varepsilon}+\hat{V}_{B \varepsilon}$

enquanto a eq. (5), cujo operador Hamiltoniano é da forma

$$
\hat{H}_{N}=\hat{T}_{A}+\hat{T}_{B}+\hat{V}_{A B}+W_{n}\left(\stackrel{r}{r_{A B}}\right)
$$

permite descrever o movimento dos núcleos, sujeitos a um potencial efetivo devido ao movimento eletrônico. É importante observar que a eq. (5) só poderá ser resolvida quando conhecermos a função $W_{n}\left(\dot{r}_{A B}\right)$, ou seja, após resolvermos a eq. (4) para várias configurações dos núcleos. Os termos $V_{A B}+W_{n}\left(\vec{r}_{A B}\right)=U_{n}\left(\vec{r}_{A B}\right)$ representam o potencial efetivo total ao qual os núcleos estão submetidos. No caso de moléculas poliatômicas, a função $U_{n}\left(\stackrel{1}{r}_{A B}\right)$ é denominada superfície ou hipersuperfície de energia potencial da molécula ${ }^{19,20}$. Agora, temos dois problemas a serem analisados e que podem ser estudados separadamente: um relacionado ao espectro eletrônico, dado pela eq. (4), e outro relacionado ao espectro nuclear, dado pela eq. (5).

\section{O ESPECTRO ELETRÔNICO}

Assumindo-se a aproximação de Born-Oppenheimer o problema eletrônico do $\mathrm{H}_{2}{ }^{+}$fica reduzido a um problema de um corpo $^{12,14}$, que possui solução exata. Para solucioná-lo devemos usar um sistema de coordenadas apropriado, i.e., que leve em consideração a simetria da molécula. Neste caso podemos escolher um sistema de coordenadas elípticas ${ }^{12,15,16}$, o qual é definido a partir das relações:

$\mu=\frac{r_{A}+r_{B}}{r_{A B}} ; \quad v=\frac{r_{A}-r_{B}}{r_{A B}} ; \phi=\phi$

onde $r_{A} r_{B} r_{A B}$ e $\phi$ são variáveis definidas como na Figura 1. O elemento de volume neste sistema é dado por

$d \tau=\frac{r_{A B}^{3}}{8}\left(\mu^{2}-v^{2}\right) d \mu d v d \phi$

sendo os limites de integração para as variáveis $\mu: 1$ a $\infty, v$ : -1 a $1, \phi: 0$ a $2 \pi$. As superfícies $\mu=$ constante definem elipsóides de revolução, com os núcleos nos focos, e as superfícies $v=$ constante definem hiperbolóides confocais.

Então, escrevendo a eq. (4) no sistema de coordenadas elípticas, obtemos uma equação diferencial parcial de segunda ordem, i.e.,

$$
\begin{aligned}
& \left\{-\frac{h^{2}}{2 m_{\varepsilon}} \frac{4}{r_{A B}^{2}\left(\mu^{2}-v^{2}\right)}\left[\frac{\partial}{\partial \mu}\left(\left(\mu^{2}-1\right) \frac{\partial}{\partial \mu}\right)+\frac{\partial}{\partial v}\left(\left(1-v^{2}\right) \frac{\partial}{\partial v}\right)\right.\right. \\
& \left.+\frac{\mu^{2}-v^{2}}{\left(\mu^{2}-1\right)\left(1-v^{2}\right)} \frac{\partial^{2}}{\partial \phi^{2}}\right]-\frac{4 e^{2}}{r_{A B}} \frac{\mu}{\mu^{2}-v^{2}} \\
& \left.+\frac{e^{2}}{r_{A B}}\right\} \psi_{n}(\mu, v, \phi)=W_{n} \psi_{n}(\mu, v, \phi)
\end{aligned}
$$

a qual é separável em três equações diferenciais ordinárias de segunda ordem ${ }^{21}$, quando escrevemos $\psi(\mu, v, \phi)=M(\mu) N(v) \Phi(\phi)$ :

$$
\frac{d^{2} \Phi_{m}}{d \phi^{2}}=-m^{2} \Phi_{m}
$$

$\left[-\frac{d}{d v}\left(\left(1-v^{2}\right) \frac{d}{d v}\right)+\frac{m^{2}}{1-v^{2}}+\frac{D^{2} W^{\prime}}{4} v^{2}\right] N_{k}=-k N_{k}$

$\left[-\frac{d}{d \mu}\left(\left(\mu^{2}-1\right) \frac{d}{d \mu}\right)+\frac{m^{2}}{\mu^{2}-1}-2 D \mu-\frac{D^{2} W^{\prime}}{4} \mu^{2}\right] M_{k}=k M_{k}$

onde usamos o raio de Bohr, $a_{0}=\mathrm{h} / m_{\varepsilon} e^{2}$, definimos a coordenada adimensional $D=r_{A B} / a_{0}$ e o termo $W^{\prime}=2\left(e^{-2} a_{0} W_{n}-D^{-1}\right)$; $k$ e $m$, são constantes de separação e as funções $M_{k}, N_{k}$ e $\Phi_{m}$ só dependem das variáveis, $\mu, v$ e $\phi$, respectivamente.

A eq. (11) reflete a simetria cilíndrica da molécula do $\mathrm{H}_{2}{ }^{+}$ em torno do seu eixo de ligação, e suas soluções normaliza$\operatorname{das}^{12,14}$ são:

$\Phi_{m}(\phi)=\frac{1}{\sqrt{2 \pi}} \mathrm{e}^{i m \phi}$ 
Uma das imposições físicas para estas soluções é que $\Phi_{m}(0)$ $=\Phi_{m}(2 \pi)$, logo o parâmetro $m$ deve ser um número inteiro, i.e., $m=0, \pm 1, \pm 2, \ldots$ Definindo-se o eixo $z$ na direção $A B$ (ver Figura 1) temos que as soluções (14) serão autofunções da componente $z$ do operador momentum angular ${ }^{12,16}$, i.e., $\hat{L}_{z} \Phi_{m}=L_{z} \Phi_{m}$. De fato, no sistema de coordenadas elípticas, esta componente assume a forma $\hat{L}_{z}=-i \mathrm{~h} \frac{\partial}{\partial \phi}$, tal que aplicada em (14) fornece

$$
\hat{L}_{z} \Phi_{m}=m h \Phi_{m}
$$

O sinal de $m$ determina o sentido de "rotação" do elétron em torno do eixo $z$. Como a energia eletrônica independe desta rotação, os estados de momentum angular orbital para um elétron ficam especificados pelo valor absoluto de $m$, ou seja, $\lambda=$ $|m|$, introduzindo-se uma degenerescência angular. Os diferentes estados de momentum angular orbital eletrônico podem ser designados conforme a Tabela 1 .

Tabela 1. Simbologia para os estados de momentum angular orbital eletrônico em moléculas diatômicas.

\begin{tabular}{crrrrr}
\hline$m$ & 0 & \pm 1 & \pm 2 & \pm 3 & $\ldots$ \\
$\lambda$ & 0 & 1 & 2 & 3 & $\ldots$ \\
estado & $\sigma$ & $\pi$ & $\delta$ & $\varphi$ & $\cdots$ \\
\hline
\end{tabular}

Os símbolos que aparecem na Tabela 1 introduzem uma nomenclatura geral para designar os estados de momentum angular de qualquer molécula diatômica. Considerando a degenerescência angular, vemos que, exceto para o estado $\sigma(m=0)$, todos os outros estados de momentum angular possuem degenerescência de segunda ordem, devido ao duplo sinal em $m$.

Apesar da discussão sobre as soluções da eq. (11) não entraremos em detalhes sobre as técnicas matemáticas para resolver as eqs. (12) e (13) (para uma análise minuciosa destes problemas deve-se consultar as referências 10, 11 e 15). Por outro lado, introduziremos um método para resolver a eq. (4) de maneira aproximada. Este método, que discutiremos a seguir, fundamenta-se no princípio variacional ${ }^{12,22}$, que é utilizado como base para o desenvolvimento de técnicas de soluções mais acuradas nos problemas que envolvem moléculas multi-eletrônicas ${ }^{22,23}$.

\section{O princípio variacional e o método LCAO*}

Consideremos a equação $\hat{H}_{\varepsilon} \psi_{n}=W_{n} \psi_{n}$, a qual descreve o problema eletrônico. Um dos métodos aproximados mais utilizados na química quântica, para a resolução deste problema, baseia-se no princípio variacional. Este princípio consiste em escrever uma função de estado tentativa dependente de certos parâmetros - os quais são determinados de forma a minimizar o valor esperado da energia do sistema em estudo - como uma solução aproximada do problema eletrônico. Isto é, seja $\psi$ uma função tentativa escrita como:

$$
\tilde{\psi}=\sum_{k}^{K} c_{k} \chi_{k}
$$

onde os $\chi_{\mathrm{k}}$ definem um conjunto de $K$ funções-base ${ }^{* *}$ e os $c_{k}$ são os coeficientes (ou parâmetros) da expansão; e seja o funcional ${ }^{22}$

$$
E[\tilde{\psi}]=\frac{\int \tilde{\psi}^{*} \hat{H} \tilde{\psi} d \tau}{\int \tilde{\psi}^{*} \tilde{\psi} d \tau}
$$

o qual define o valor esperado da energia do sistema.

\footnotetext{
* Terminologia usada na literatura, do inglês, "Linear Combination of Atomic Orbitals" .
}

O princípio variacional pode ser resumido no seguinte teorema (cuja prova não é objetivo desta comunicação):

Dado uma função de estado aproximada, satisfazendo as condições de contorno do problema, o valor esperado da energia calculado a partir desta função será sempre um limite superior da energia exata do estado fundamental.

Desta forma, o caminho usual para resolver os problemas de estrutura eletrônica molecular é escrever a função (16) como combinação linear de orbitais atômicos conhecidos ${ }^{17,18}$, e minimizar o funcional energia, $E[\psi]$, com respeito aos parâmetros da expansão. Esta é a essência da teoria do orbital molecular. Uma das motivações para o desenvolvimento desta teoria veio da suposição de que as moléculas são constituídas de átomos, e assim, é razoável pensar que a distribuição de carga eletrônica em uma molécula pode ser representada, em média, como uma soma de distribuições eletrônicas atômicas ${ }^{1,2}$.

Vamos ilustrar este método realizando os cálculos para a molécula de $\mathrm{H}_{2}{ }^{+}$e, sempre que possível, generalizá-lo para moléculas mais complexas. Notemos, no entanto, que diferentemente de uma solução matemática exata, o método variacional, em princípio, não nos permite encontrar o espectro de energia molecular completo, mas somente alguns de seus possíveis estados, uma vez que o método depende do número de funções-base utilizadas na expansão LCAO e refere-se a um estado específico do sistema. No caso particular da molécula de $\mathrm{H}_{2}{ }^{+}$, utilizaremos uma função de estado molecular composta de dois orbitais hidrogenóides tipo 1s, cada uma centrada em um dos núcleos:

$\tilde{\psi}=c_{A} \chi_{A}+c_{B} \chi_{B}$

sendo $\chi_{k}=\sqrt{1 / \pi a_{0}^{3}} \mathrm{e}^{-r_{k} / a_{0}},{ }_{k=A, B}$, funções de estado normalizadas para o átomo de hidrogênio ${ }^{12,14}$.

De acordo com (17), o valor esperado da energia eletrônica será dado por

$W=\frac{\int \tilde{\psi}^{*} \hat{H}_{\varepsilon} \tilde{\psi} d \tau}{\int \tilde{\psi}^{*} \tilde{\psi} d \tau}$

Então, substituindo (18) em (19), temos

$W=\frac{c_{A}^{2} \int \chi_{A}^{*} \hat{H}_{\varepsilon} \chi_{A} d \tau+2 c_{A} c_{B} \int \chi_{A}^{*} \hat{H}_{\varepsilon} \chi_{B} d \tau+c_{B}^{2} \int \chi_{B}^{*} \hat{H}_{\varepsilon} \chi_{B} d \tau}{c_{A}^{2} \int \chi_{A}^{*} \chi_{A} d \tau+2 c_{A} c_{B} \int \chi_{A}^{*} \chi_{B} d \tau+c_{B}^{2} \int \chi_{B}^{*} \chi_{B} d \tau}$

Usando o fato de $\chi_{A}$ e $\chi_{B}$ serem normalizadas e introduzindo a notação

$H_{\mu v}=\int \chi_{\mu}^{*} \hat{H}_{\varepsilon} \chi_{v} d \tau$

$S_{\mu v}=\int \chi_{\mu}^{*} \chi_{v} d \tau$

a eq. (20) torna-se

$W=\frac{c_{A}^{2} H_{A A}+2 c_{A} c_{B} H_{A B}+c_{B}^{2} H_{B B}}{c_{A}^{2}+2 c_{A} c_{B} S_{A B}+c_{B}^{2}}$

Agora, devemos minimizar a eq. (22) com respeito aos parâmetros $c_{A}$ e $c_{B}$, i.e., devemos resolver as equações

\footnotetext{
** Entende-se por funções-base um conjunto de funções linearmente independentes, pertencentes a um dado espaço, e suficientes para expandir qualquer função genérica do mesmo espaço. Em química quântica é comum utilizar-se funções-base tipo gaussiana (GTOs), orbitais de Slater (STOs) ou orbitais hidrogenóides.
} 
$\left(\frac{\partial W}{\partial c_{A}}\right)_{C_{B}}=0 ;\left(\frac{\partial W}{\partial c_{B}}\right)_{C_{A}}=0$

Depois de algumas manipulações algébricas obtemos o seguinte sistema de equações:

$c_{A}\left(H_{A A}-W\right)+c_{B}\left(H_{A B}-S_{A B} W\right)=0$

$c_{A}\left(H_{B A}-S_{A B} W\right)+c_{B}\left(H_{B B}-W\right)=0$

As eqs. (24-a) e (24-b) são conhecidas como equações seculares. A condição para que um sistema de equações desta natureza tenha soluções não triviais (i.e., outras soluções além de $c_{A}=c_{B}=0$ ), é que o determinante dos coeficientes se anule. Logo, para o sistema de equações (24), deve ser verdade que

$\left|\begin{array}{lr}H_{A A}-W & H_{A B}-S_{A B} W \\ H_{B A}-S_{A B} W & H_{B B}-W\end{array}\right|=0$

Como o Hamiltoniano (6) tem simetria cilíndrica, ou seja, é invariante por rotações em torno do eixo $z$ (ver Figura 1), e as funções $\chi_{\kappa}$ são idênticas, podemos simplificar o determinante (25) notando que $H_{A A}=H_{B B}$ e $H_{A B}=H_{B A}$, o que nos dá

$\left(H_{A A}-W\right)^{2}-\left(H_{A B}-S_{A B} W\right)^{2}=0$

isto é,

$H_{A A}-W= \pm\left(H_{A B}-S_{A B} W\right)=0$

O determinante secular (25) nos leva a uma equação característica (um polinômio de segundo grau em $W$ ) que fornece duas raízes, ambas maiores que a energia eletrônica exata da molécula. Assim, de (27) obtemos

$W_{s}=\frac{H_{A A}+H_{A B}}{1+S_{A B}}$

$W_{a}=\frac{H_{A A}-H_{A B}}{1-S_{A B}}$

Substituindo as eqs. (28-a) e (28-b), uma de cada vez, no sistema de eqs. (24) obtemos os coeficientes da expansão LCAO, i.e.,

$c_{A}^{s}=c_{A}^{s}$

$c_{A}^{a}=-c_{B}^{a}$

os quais permitem determinar duas funções de estado ou orbitais moleculares ${ }^{*}$, que normalizados são

$\psi_{s}=\frac{1}{\sqrt{2+2 S_{A B}}}\left(\chi_{A}+\chi_{B}\right)$

$\psi_{a}=\frac{1}{\sqrt{2-2 S_{A B}}}\left(\chi_{A}-\chi_{B}\right)$

\footnotetext{
Para sistemas uni-eletrônicos, orbital e estado são sinônimos, neste caso, as raízes de ordens maiores que um do determinante secular são interpretadas como limites superiores dos estados excitados. No caso de sistemas multi-eletrônicos, o estado da molécula difere dos seus orbitais moleculares (um excelente tratamento para sistemas multi-eletrônicos é dado na referência 22).
}

Os índices $s$ e $a$ referem-se a simétrico e anti-simétrico, respectivamente, uma vez que a função de estado $\psi_{s}$ é simétrica e a função de estado $\psi_{a}$ é anti-simétrica com respeito às coordenadas espaciais dos núcleos $A$ e $B$.

\section{Distribuição eletrônica e estabilidade da molécula de $\mathbf{H}_{2}^{+}$}

Até agora, vimos que o método LCAO nos permite encontrar alguns valores aproximados para os níveis de energia relacionados ao espectro molecular eletrônico, bem como os seus respectivos orbitais moleculares. É interessante, neste contexto, dar uma interpretação física para estes resultados. Para isto, de acordo com a mecânica quântica, vamos avaliar as densidades eletrônicas a partir das funções (30-a) e (30-b), considerando uma região em que $S_{A B}$ é muito pequeno, ou seja, onde

$$
\begin{aligned}
& \psi_{s}^{2}=\frac{1}{2}\left\{\chi_{A}^{2}+\chi_{B}^{2}+2 \chi_{A} \chi_{B}\right\} \\
& \psi_{a}^{2}=\frac{1}{2}\left\{\chi_{A}^{2}+\chi_{B}^{2}-2 \chi_{A} \chi_{B}\right\}
\end{aligned}
$$

Por inspeção das eqs. (31) podemos observar que o orbital molecular simétrico incrementa a densidade eletrônica na região internuclear, enquanto o orbital molecular anti-simétrico leva a um decréscimo da densidade eletrônica na mesma região. Um gráfico das densidades de carga é mostrado na Figura 2. A linha tracejada representa a densidade eletrônica dos átomos individuais, separados a uma distância infinita, e a linha cheia representa a distribuição de carga na molécula ao longo do seu eixo de ligação.
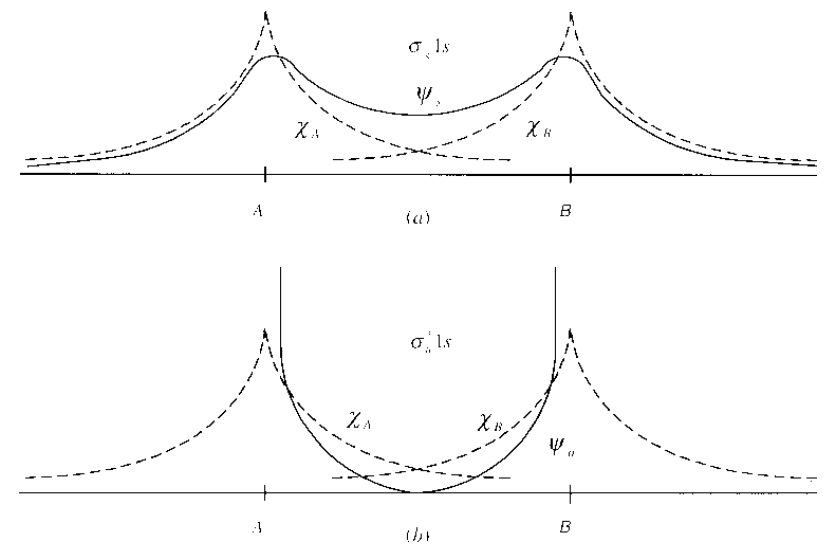

Figura 2. Densidades eletrônicas em torno do eixo internuclear do $\mathrm{H}_{2}^{+}$para os estados simétrico (a) e anti-simétrico (b). Os símbolos $\sigma_{g} 1 \mathrm{~s}$ e $\sigma_{u}{ }^{*} 1 \mathrm{~s}$ referem-se à simetria $\sigma(m=0)$, uma vez que os orbitais moleculares são provenientes da combinação de orbitais atômicos tipo $s$, cujo momentum angular é nulo; o indice g (do alemão "gerade") indica que a função $\psi_{s}$ é par e o índice u (do alemão "ungerade") indica a função $\psi_{a}$ é ímpar; e o sinal "*" denota uma superposição destrutiva das funções de estado atômicas.

A partir desta análise podemos introduzir a idéia de orbital ligante - o qual favorece uma concentração de carga entre os núcleos - e orbital anti-ligante - o qual tende a diminuir a densidade de carga na região internuclear, concentrando-a sobre os núcleos. De fato, o orbital ligante contribui para a formação de uma ligação covalente na molécula de $\mathrm{H}_{2}{ }^{+}$originando, conseqüentemente, uma espécie estável. Este fato requer que a energia eletrônica do estado simétrico, $W_{s}$, tenha um mínimo para um certo valor da separação internuclear (definido como distância de equilíbrio) da molécula.

Podemos obter informações quantitativas sobre os estados $W_{s}$ e $W_{a}$ em função da distância internuclear do $\mathrm{H}_{2}{ }^{+}$se avaliarmos as integrais $H_{A A}, H_{A B}$ e $S_{A B}$. Assim, usando a equação ${ }^{12}$ 
$\left[-\frac{\mathrm{h}}{2 m_{\varepsilon}} \nabla_{\varepsilon}^{2}-\frac{e^{2}}{r_{A}}\right] \chi_{A}=W_{H} \chi_{A}$

juntamente com as eqs. (6), (21-a) e (21-b), obtemos

$H_{A A}=\int \chi_{A}\left(W_{H}-\frac{e^{2}}{r_{B}}\right) \chi_{A} d \tau=W_{H}+J$

onde a integral $J$ pode ser resolvida, por partes, em coordenadas elípticas, isto é,

$J=-\frac{D^{2} e^{2}}{4 \pi a_{0}} \int_{1-1}^{\infty} \int_{1}^{1} \int_{0}^{2 \pi} e^{-D(\mu+v)}(\mu+v) d \mu d v d \phi=-\frac{e^{2}}{a_{0}}\left\{-\frac{1}{D}+e^{-2 D}\left(1+\frac{1}{D}\right)\right\}$

As integrais $H_{A B}$ e $S_{A B}$ são calculadas de maneira similar,

$H_{A B}=\int \chi_{A}\left(W_{H}-\frac{e^{2}}{r_{B}}\right) \chi_{B} d \tau=S_{A B} W_{H}+K$

para a qual temos

$S_{A B}=\frac{D^{3}}{8 \pi} \int_{1-1}^{\infty} \int_{-1}^{1} \int_{0}^{2 \pi} \mathrm{e}^{-D \mu}\left(\mu^{2}-v^{2}\right) d \mu d v d \phi=\mathrm{e}^{-D}\left(1+D+1 / 3 D^{2}\right)$

$\mathrm{e}$

$K=-\frac{D^{2} e^{2}}{4 \pi a_{0}} \int_{1-10}^{\infty} \int_{-1}^{12 \pi} \mathrm{e}^{-D}(\mu+v) d \mu d v d \phi=-\frac{e^{2}}{a_{0}} \mathrm{e}^{-2 D}(1+D)$

onde usamos a coordenada adimensional $D=r_{A B} / a_{0}$.

É muito comum, na literatura, dá-se uma interpretação física às integrais $S_{A B}, J$ e $K$, no âmbito da teoria do orbital molecular: $S_{A B}$ representa a superposição entre dois orbitais atômicos centrados em núcleos distintos e é definida como integral de recobrimento ou overlap; $J$, denominado integral coulombiana, representa a interação eletrostática entre a densidade de carga eletrônica no orbital atômico $A$ e a carga do núcleo $B$; e $K$, definida como integral de troca ou exchange, representa a energia de troca, devido ao fato do elétron não pertencer a nenhum dos átomos, mas sim à molécula como um todo ${ }^{12,16,22}$.

Com os resultados (33), (35) e (36) é possível determinar as energias eletrônicas, eqs. (28-a) e (28-b) em função da distância internuclear na molécula, ou seja,

$W_{s}=W_{H}+\frac{J+K}{1+S_{A B}}$

$\mathrm{e}$

$W_{a}=W_{H}+\frac{J-K}{1-S_{A B}}$

Alguns valores destas energias, para os estados $\sigma_{\mathrm{g}} 1 \mathrm{~s}$ e $\sigma_{u}{ }^{*} 1 s$, são apresentados na Tabela 2 como função da distância internuclear.

A partir dos valores de $W_{s}, W_{a}$ e $1 / r_{A B}$ podemos construir o diagrama de energia potencial, $U\left(r_{A B}\right)=W\left(r_{A B}\right)+1 / r_{A B}$, da molécula de $\mathrm{H}_{2}^{+}$(Figura 3), e logo, determinar sua distância de equilíbrio e a energia de dissociação* .

* A energia de dissociação de uma molécula diatômica é definida como o valor absoluto da diferença entre sua energia potencial, quando os átomos estão na posição de equilíbrio, e a energia eletrônica dos átomos separados a uma distância infinita.
Tabela 2. Energias eletrônicas calculadas (em unidades de $e^{2} / a_{0}$ ) para os estados simétrico e anti-simétrico da molécula de $\mathrm{H}_{2}{ }^{+}$ com respeito à coordenada adimensional $D=r_{A B} / a_{0}$.

\begin{tabular}{lll}
\hline $\mathrm{D}$ & $-W_{\mathrm{s}}$ & $-W_{\mathrm{a}}$ \\
\hline 1,0 & 1,288 & 0,455 \\
1,4 & 1,186 & 0,574 \\
1,8 & 1,096 & 0,640 \\
2,0 & 1,054 & 0,661 \\
2,4 & 0,981 & 0,687 \\
2,8 & 0,919 & 0,698 \\
3,2 & 0,867 & 0,702 \\
3,6 & 0,824 & 0,699 \\
4,0 & 0,787 & 0,695 \\
5,0 & 0,719 & 0,676 \\
6,0 & 0,676 & 0,657 \\
7,0 & 0,647 & 0,639 \\
8,0 & 0,627 & 0,623 \\
$\infty$ & 0,500 & 0,500 \\
\hline
\end{tabular}

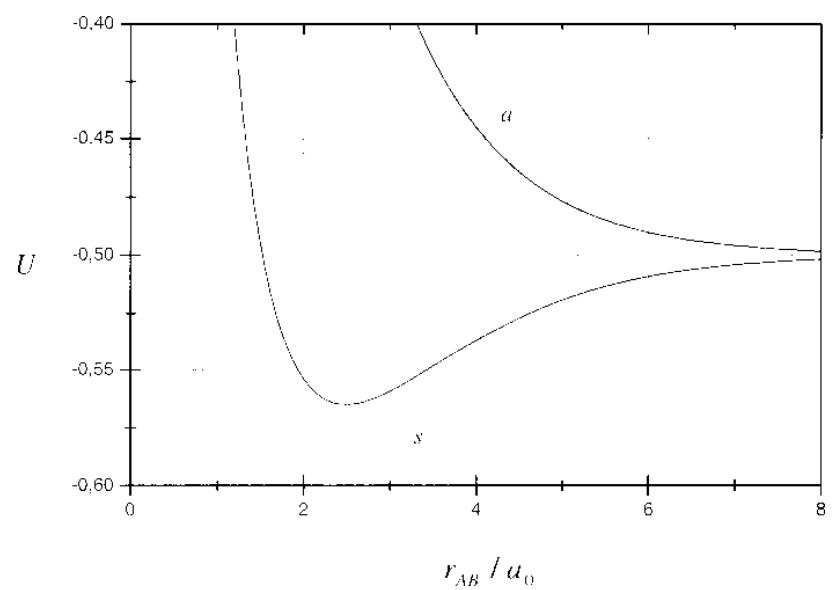

Figura 3. Curvas de energia potecial (em unidades de $e^{2} / a_{0}$ ) como função da distância internuclear da molécula de $\mathrm{H}_{2}^{+}$para os estados simétrico (s) e anti-simétrico (a). A energia do estado simétrico possui um ponto de mínimo, o que favorece a formação de uma molécula estável.

Os valores da energia de dissociação e da distância de equilíbrio, calculados na aproximação LCAO, utilizando-se uma expansão de duas funções-base tipo hidrogenóide para a molécula de hidrogênio ionizada são de $1,76 \mathrm{eV}$ e 1,32 $\AA$, respectivamente. Estes resultados, quando comparados aos correspondentes dados experimentais $^{24}(2,791 \mathrm{eV}$ e $1,060 \AA)$ concordam grosseiramente, uma vez que os orbitais hidrogenóides não consideram o efeito de polarização do átomo de hidrogênio no campo do íon $\mathrm{H}^{+}$. Entretanto, é possível introduzir outros parâmetros variacionais na função (18), como por exemplo um fator de carga nuclear efetiva ${ }^{12}$, de modo que esses resultados possam ser melhorados satisfatoriamente. Neste caso, os valores calculados são 2,25 eV para a energia de dissociação e 1,06 $\AA$ para a separação de equilíbrio. O uso de funções de polarização na expansão LCAO também é discutido na referência 12, p 331; tais funções permitem um cálculo mais acurado das propriedades moleculares do $\mathrm{H}_{2}{ }^{+}$.

\section{O ESPECTRO NUCLEAR}

Concentramos nossa atenção, até agora, no estudo do movimento eletrônico sob o potencial dos núcleos fixos. A etapa seguinte de nossa análise é considerar o movimento nuclear, 
descrito pela eq. (5). Para efeito de simplificação vamos transformar o problema dos dois núcleos em um problema de um corpo. Isto pode ser feito escolhendo-se um sistema de coordenadas que considera o centro de massa e as variáveis internas, $r, \theta$, e $\varphi$, de um núcleo em relação ao outro (Figura 4).

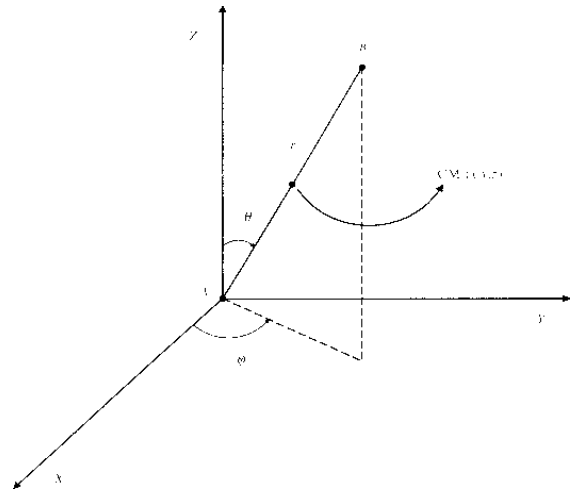

Figura 4. Sistema de coordenadas para o movimento interno da molécula de $\mathrm{H}_{2}{ }^{+}$. As coordenadas do centro de massa, CM, referem-se ao movimento translacional da molécula.

Para o sistema de coordenadas da Figura 4, a função de estado dos núcleos, $X_{n, v}$, pode ser escrita como

$$
X_{n, v}\left(\stackrel{\perp}{r_{A B}}\right)=X_{n, v}^{C M}(x, y, z) \chi_{n, v}(r, \theta, \varphi)
$$

onde $X_{n, v}^{C M}$ é a função de estado associada ao movimento translacional do centro de massa da molécula e a função de estado $\chi_{n, v}$ está associada aos movimentos internos de rotação e vibração dos núcleos ${ }^{12}$. Assim, em termos das coordenadas esféricas, $(r, \theta, \varphi)$, a eq. (5) pode ser reescrita como

$$
\begin{aligned}
& \left\{-\frac{h^{2}}{2 \mu}\left[\frac{1}{r^{2}} \frac{\partial}{\partial r}\left(r^{2} \frac{\partial}{\partial r}\right)+\frac{1}{r^{2} \operatorname{sen} \theta} \frac{\partial}{\partial \theta}\left(\operatorname{sen} \theta \frac{\partial}{\partial \theta}\right)+\right.\right. \\
& \left.\left.\frac{1}{r^{2} \operatorname{sen}^{2} \theta} \frac{\partial^{2}}{\partial \varphi^{2}}\right]+U_{n}(r)\right\} \chi_{n, v}=E_{n, v} \chi_{n, v}
\end{aligned}
$$

sendo $\mu=\frac{m_{A} m_{B}}{m_{A}+m_{B}}$ a massa reduzida do sistema e $U_{n}(r)=V_{A B}$ $+W_{n}(r)$ a energia potencial à qual os núcleos estão submetidos.

A eq. (39), assim como a eq. (10), também pode ser separada em três equações diferenciais de segunda ordem, se escrevermos $\chi(r, \theta, \varphi)=R(r) \Theta(\theta) \Phi(\varphi)$, ou seja,

$$
\frac{d^{2} \Phi_{M}}{d \varphi^{2}}=-M^{2} \Phi_{M}
$$

$$
\left[-\frac{1}{\operatorname{sen} \theta} \frac{d}{d \theta}\left(\operatorname{sen} \theta \frac{d}{d \theta}\right)+\frac{M^{2}}{\operatorname{sen}^{2} \theta}\right] \Theta_{J, M}(\theta)=J(J+1) \Theta_{J, M}(\theta)
$$

$$
\left\{-\frac{h^{2}}{2 \mu}\left[\frac{1}{r^{2}} \frac{d}{d r}\left(r^{2} \frac{d}{d r}\right)-\frac{J(J+1)}{r^{2}}\right]+U_{n}(r)\right\} R_{n}(r)=E_{n, v} R_{n}(r)
$$

As soluções de (40) são semelhantes às soluções da eq. (11), i.e.,

$\Phi_{M}(\varphi)=\frac{1}{\sqrt{2 \pi}} \mathrm{e}^{i M \varphi}$ porém, neste caso, elas estão associadas à componente $\mathrm{Z}$ (ver Figura 4) do momentum angular de rotação da molécula, de forma que podemos fazer uma análise similar à do momentum angular eletrônico. Já as soluções da eq. (41) são da forma

$\Theta_{J, M}(\theta)=\left\{\frac{(2 J+1)(J-|M|) !}{2(J+|M|) !}\right\}^{1 / 2} P_{M}^{|J|}(\cos \theta)$

sendo $P_{M}^{|J|}(\cos \theta)$, os polinômios associados de Legendre ${ }^{12,14}$.

As funções de estado correspondentes ao movimento rotacional são os harmônicos esféricos, definidos como

$Y_{J, M}(\theta, \varphi)=\Theta_{J, M}(\theta) \Phi_{M}(\varphi)$

onde os índices $J$ e $M$, por razões físicas, devem assumir os valores $J=0,1,2, \ldots ; M=-J,-J+1, \ldots, J-1, J$. Estes números estão relacionados com o momentum angular de rotação da molécula (para uma descrição detalhada ver referências $12, \mathrm{p}$ 265; e 14, p 23).

Os valores das energias associadas aos estados rotacionais, eq. (45), podem ser obtidos em primeira aproximação, considerando os núcleos à distância de equilíbrio $r_{e}$ como um rotor rígido. Assim, temos que a energia cinética de rotação fica dada em termos do operador momentum angular rotacional, $\boldsymbol{L}_{J}$, e do momentum de inércia $I_{e}=\mu r_{e}^{2}$, i.e., $\hat{T}_{J}=\hat{\boldsymbol{L}}_{J}^{2} / 2 I_{e}$. Em virtude da quantização do momentum angular podemos expressar as energias rotacionais como

$$
E_{J}=\frac{h^{2}}{2 I_{e}} J(J+1)=B_{e} h c J(J+1)
$$

onde $B_{e}$ é definido como a constante rotacional da molécula ${ }^{26}$.

\section{A natureza da função $U_{n}(r)$}

As soluções da eq. (42) dependem da forma funcional da energia eletrônica, $W_{n}(r)$. Em princípio, poderíamos utilizar as funções $W_{s}$ ou $W_{a}$, determinadas pelo método LCAO. Por outro lado, existem algumas funções menos complicadas, para as quais esta equação tem soluções exatas. Discutiremos a seguir uma das formas mais simples para $U_{n}(r)$. Uma análise da Figura 5 nos mostra que, para regiões próximas do equilíbrio, esta função tem uma tendência oscilatória e, portanto, é razoável modelar o movimento dos dois núcleos como o movimento de um oscilador harmônico ${ }^{12,25,26}$.

Vamos considerar que os núcleos da molécula de $\mathrm{H}_{2}{ }^{+}$estejam submetidos a um potencial do tipo harmônico, i.e.,

$U(r)=\frac{1}{2} k\left(r-r_{e}\right)^{2}$

onde $k$ é a constante de força interatômica. Este modelo é claramente limitado às regiões em que os núcleos estão muito próximos da posição de equilíbrio, de modo que não podemos utilizá-lo para estudar a dissociação molecular, uma vez que o comportamento assintótico do potencial harmônico não corresponde ao potencial vibracional exato (ver Figura 5).

$\mathrm{Se}$ o movimento rotacional for ignorado, i.e., $J=0$, e fizermos $R(r)=S(r) / r$ na eq. (42), com o potencial (47), reduziremos o movimento vibracional ao movimento de um oscilador harmônico unidimensional ${ }^{25}$. Este problema tem soluções exatas e fornece a seguinte expressão para os níveis de energia vibracional

$$
E_{v}=h v_{e}\left(v+\frac{1}{2}\right)=h c \omega_{e}\left(v+\frac{1}{2}\right)
$$




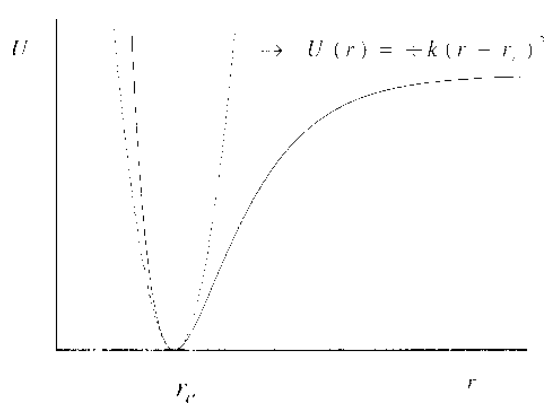

Figura 5. Comparação da curva de potencial típica para uma molécula diatômica com o potencial de um oscilador harmônico.

onde $v_{e}=(1 / 2 \pi)(k / \mu)^{1 / 2}$ e $v$ é o número quântico vibracional, que pode tomar valores $v=0,1,2, \ldots$.

As soluções da eq. (42) tornam-se mais complicadas quando a contribuição rotacional é levada em consideração. Uma descrição para este caso é dada por Iachello e Levine ${ }^{26}$, que mostram a seguinte expressão aproximada para os níveis de energia roto-vibracionais

$E_{v, J}=h c\left[\omega_{e}\left(v+\frac{1}{2}\right)+B_{e} J(J+1)-G_{e} J^{2}(J+1)^{2}\right]$

sendo $B_{e}$ a constante rotacional de equilíbrio, eq. (46), da molécula

$B_{e}=\frac{h}{8 \pi^{2} c I_{e}}$

e $G_{e}$, a constante de distorção centrífuga, definida em função da constante rotacional $B_{e}$ e da freqüêencia angular $\omega_{e}$, pela expressão

$$
G_{e}=4 B_{e}^{3} / \omega_{e}^{2}
$$

O modelo harmônico, entretanto, prediz que os níveis de energia vibracional são igualmente espaçados. Por outro lado, os experimentos indicam que a separação entre sucessivos níveis decresce com o número quântico $v$. O modelo do oscilador, portanto, é adequado para analisar as propriedades moleculares quando os núcleos estão próximos às suas posições de equilíbrio.

\section{CONCLUSÕES}

Neste trabalho, procurou-se dar uma visão geral da moderna teoria de estrutura molecular abordando os principais aspectos do seu formalismo no âmbito da teoria quântica. Para isto, partiu-se de um problema simples, envolvendo a molécula de hidrogênio ionizada, estabelecendo-se as aproximações adequadas para a separação dos espectros eletrônico e nuclear. Finalmente, realizou-se cálculos simplificados a fim de ilustrar o funcionamento dos métodos de aproximação, bem como verificar sua validade.

\section{AGRADECIMENTOS}

Os autores agradecem ao professor J. D. M. Vianna pelas discussões e sugestões e à CAPES pelo suporte financeiro.

\section{REFERÊNCIAS}

1. Bader, R. F. W. In Atoms in Molecules; Claredon Press Oxford; New York, 1990.

2. Bader, R. F. W.; Popelier, P. L. A.; Keith, T. A.; Angew. Chem. Ed. Engl. 1994, 33, 620.

3. Hylleraas, E. A.; Z. Phys. 1928, 48, 469.

4. Hylleraas, E. A.; Z. Phys. 1928, 51, 150.

5. Hylleraas, E. A.; Z. Phys. 1929, 54, 347.

6. Morse, P. M.; Stueckelberg, E. C. G.; Phys. Rev. 1929, 33, 932.

7. Lennard-Jones, J. E.; Trans. Faraday Soc. 1929, 24, 668.

8. Teller, E.; Z. Phys. 1930, 61, 468.

9. Eckart, C.; Phys. Rev. 1930, 36, 878.

10. Hylleraas, E. A.; Z. Phys. 1931, 71, 739.

11. Jaffé, G.; Z. Phys. 1934, 87, 535.

12. Pauling, L.; Wilson, E. B. In Introduction to Quantum Mechanics; McGraw-Hill; New York, 1935.

13. Peixoto, E. M. A.; Quim. Nova 1978, 1(1), 5.

14. Peixoto, E. M. A.; Quim. Nova 1978, 1(2), 10.

15. Bates, D. R.; Ledshman, K.; Stwart, A. L.; Phil. Trans. Roy. Soc. A 1953, 246, 215.

16. Hanna, M. W. In Quantum Mechanics in Chemistry; W. A. Benjamin; New York, 1969.

17. Roothaan, C. C. J.; Rev. Mod. Phys. 1951, 23, 69.

18. Cioslowski, J.; Surján, P. R.; J. Mol. Struct. (Theochem) 1992, 9, 255.

19. Wilson, S. In Electron Correlation in Molecules; Claredon Press Oxford; New York, 1984.

20. Laidler, K. J. In Theories of Chemical Reaction Rates; McGraw-Hill; New York, 1969.

21. Ley-Koo, E.; Cruz, S. A.; J. Chem. Phys. 1981, 74, 4603.

22. McWeeny, R. In Methods of Molecular Quantum Mechanics; Academic Press; San Diego, 1992.

23. Morgon, N. H.; Custódio, R.; Quim. Nova 1995, 18, 44.

24. Herzberg, G. In Molecular Spectra and Molecular Structure; Van Nostrand Reinhold; New York, 1950.

25. Peixoto, E. M. A.; Quim. Nova 1978, 1(3), 13.

26. Iachello, F.; Levine, R. D. In Algebraic Theory of Molecules; Oxford University Press; New York, 1995. 